\title{
NONSTATIONARY BLIND IMAGE RESTORATION USING VARIATIONAL METHODS
}

\author{
Tom E. Bishop ${ }^{a}$, Rafael Molina ${ }^{b}$, James R. Hopgood $^{a}$ \\ a) IDCOM, Joint Research Institute for Signal \& Image Processing, \\ School of Engineering \& Electronics, The University of Edinburgh, Edinburgh, EH9 3JL, UK \\ b) Dept. Ciencias de la Computación e I. A., Univ. de Granada, 18071 Granada, Spain. \\ t.e.bishopded.ac.uk, rmsddecsai.ugr.es, james.hopgoodded.ac.uk
}

\begin{abstract}
The Variational Bayesian approach has recently been proposed to tackle the blind image restoration (BIR) problem. We consider extending the procedures to include realistic boundary modelling and non-stationary image restoration. Correctly modelling the boundaries is essential for achieving accurate blind restorations of photographic images, whilst nonstationary models allow for better adaptation to local image features, and therefore improvements in quality.
\end{abstract}

Index Terms - Blind Deconvolution, Boundary modelling, Nonstationary Image models, Variational Bayesian methods

\section{INTRODUCTION}

In blind image restoration (BIR) we are concerned with estimating both the original image from a blurred observation, and the blur that caused it. Often we also wish automatic estimation of model parameters controlling the restoration. When the degree of uncertainty is great, as here, it is necessary to impose as much structure as possible representing our prior beliefs to give meaningful solutions to this ill-posed problem. This may be done robustly and effectively with a hierarchical Bayesian model.

In the Bayesian framework we introduce prior models for the unknown parameters, which in turn depend on hyperparameters. A further stage in the hierarchical Bayesian framework is to regard the hyperparameters as unknown variables and model our prior knowledge of their distributions with hyperpriors. This abstraction allows for greater robustness of estimates when we are less confident in the observations and the hyperparameters themselves.

Under this framework several restoration options may be considered; a full review of BIR methods may be found in [1]. In this work we consider using the variational Bayesian (VB) approach; this has been recently applied to the BIR problem [2], using a stationary simultaneous autoregression (SAR) model. However, real photographic images clearly contain local features; investigations suggest that using a nonstationary model both aids accurate blur identification [3], and improves the restored image [4]. In this work we consider both aspects using the VB approach.

Furthermore, to deal correctly with blurred photographic images, we use an observation model that takes into account boundary effects. These have long plagued image restoration of natural scenes, with a variety of ad-hoc schemes devised to combat the ringing artefacts at the borders that they introduce. Whilst these schemes may visually suppress the artefacts, in an iterative BIR setting they can hinder correct estimation of the blur. These artefacts occur due to objects outside the observed image canvas being convolved into the blurred image (hence these problems are often negligible in astronomical and medical imaging, but critical for photographs). Here

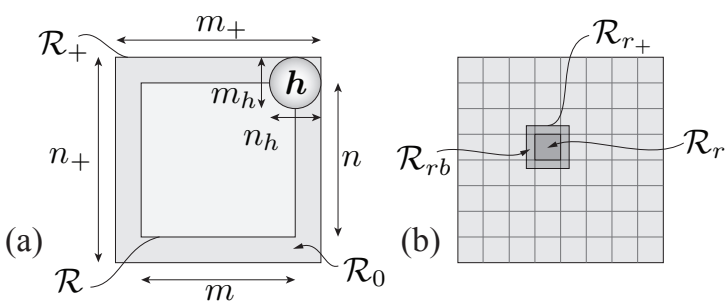

Fig. 1. (a) The extended image canvas $\mathcal{R}_{+}$, boundary region $\mathcal{R}_{0}$, and observation region $\mathcal{R}$. $\mathcal{R}_{+}$is of size $\left\{m_{+}, n_{+}\right\}=\left\{m+m_{h}-\right.$ $\left.1, n+n_{h}-1\right\}$, since the PSF is assumed to be zero outside a support of size $m_{h} \times n_{h}$. (b) A segmentation of $\mathcal{R}_{+}$, with a region $\mathcal{R}_{r}$, its border pixels $\mathcal{R}_{r b}$, and their union $\mathcal{R}_{r_{+}}$shown.

we follow the full Bayesian treatment by incorporating them into the extended observation model (see [5] for a similar scheme).

We begin in $\$ 2$ by describing the problem and the models proposed to tackle it. In $\$ 3$ we describe the VB approach to parameter estimation, and in 4 its application to the models used. In order to incorporate the boundary model and nonstationarity, we describe how the implement the iterative VB approach in the spatial domain in $\$ 5$. Results and conclusions are given in $\$ 6$ and $\$ 7$

\section{PROBLEM FORMULATION}

The BIR problem consists of estimating an unobserved true image, $f(i, j)$, and blur or point-spread function (PSF), $h(i, j)$ from an observed degraded image $g(i, j)$, which is modelled as a discrete convolution $f(i, j) * h(i, j)$ plus additive white Gaussian noise (WGN), $w(i, j)$. In matrix-vector form this may be written as

$$
\boldsymbol{g}=\boldsymbol{H}_{+} \boldsymbol{f}_{+}+\boldsymbol{w}
$$

where $\quad \boldsymbol{H}_{+} \boldsymbol{f}_{+}=\left[\boldsymbol{H} \mid \boldsymbol{H}_{0}\right]\left[\frac{\boldsymbol{f}}{\boldsymbol{f}_{0}}\right]=\boldsymbol{H} \boldsymbol{f}+\boldsymbol{H}_{0} \boldsymbol{f}_{0}$.

The extended observation model used here is shown in Fig. 1 . where the original image $f_{+}$is defined on a canvas $\mathcal{R}_{+}$and is partitioned into $f$, defined on the observation region $\mathcal{R}$, and $f_{0}$, defined on the boundary region $\mathcal{R}_{0}$. The pixels in $\boldsymbol{f}_{0}$ influence $\boldsymbol{g}$ through the blurring operation, although $\boldsymbol{g}$ is only defined on $\mathcal{R}$. The matrix $\boldsymbol{H}_{+}$ is partitioned accordingly. Note we may also write $\boldsymbol{H}_{+} \boldsymbol{f}_{+}=\boldsymbol{F}_{h} \boldsymbol{h}$.

Two options are available for the restoration. Firstly, we may estimate the extended image from $\mathrm{p}\left(\boldsymbol{f}_{+} \mid \boldsymbol{g}, \cdot\right)$, which corresponds to extrapolation based on the available data. Secondly, we may marginalise the boundary pixels $f_{0}$, i.e. we estimate just $f$ using $\mathrm{p}(\boldsymbol{f} \mid \boldsymbol{g}, \cdot) \propto \int_{\boldsymbol{f}_{0}} \mathrm{p}\left(\boldsymbol{f}_{+} \mid \boldsymbol{g}, \cdot\right) \mathrm{d} \boldsymbol{f}_{0}$. This accounts for the uncertainty of $\boldsymbol{f}_{0}$, rather than assuming it is zero as most methods do. Both 
options are considered in [5], and although marginalisation is strictly more favourable, the computational demands are much higher.

Therefore we simply estimate the extended image and discard the border region, which gives satisfactory results in practice. This simply requires using the extended $\boldsymbol{H}_{+}$of size $m n \times m_{+} n_{+}$. Based on the WGN assumption, $\boldsymbol{w} \sim \mathcal{N}\left(\mathbf{0}, \sigma_{w}^{2} \boldsymbol{I}\right)$, the likelihood of the observations conditioned on the true image may then be written

$$
\mathrm{p}\left(\boldsymbol{g} \mid \boldsymbol{f}_{+}, \boldsymbol{h}, \sigma_{w}^{2}\right)=\mathcal{N}\left(\boldsymbol{g} \mid \boldsymbol{H}_{+} \boldsymbol{f}_{+}, \sigma_{w}^{2} \boldsymbol{I}\right) .
$$

\subsection{Nonstationary Image Model and Blur Model}

Nonstationary models have been shown to improve restorations of the image, and aid in blur identification. Previously, a block-stationary AR (BSAR) model has been used in [3] to represent the image. Here we use a model that is a compromise between the more adaptable but computationally complex BSAR model, and the simpler SAR model in [2]; the image is partitioned into regions which each have their own excitation variance, but the autoregressive (AR) model parameters are fixed (using the SAR model). A fixed segmentation of the image is currently used, but we are working on tackling the BIR and segmentation problems jointly using the Bayesian framework.

The AR model may be written as $\boldsymbol{f}_{+}=\boldsymbol{A} \boldsymbol{f}_{+}+\boldsymbol{v}$, where $\boldsymbol{v} \sim \mathcal{N}\left(\mathbf{0}, \boldsymbol{Q}_{v}\right)$ is the excitation signal representing local image activity, $\boldsymbol{Q}_{v}$ is a diagonal matrix parameterised by the excitation variances $\boldsymbol{\sigma}_{v}$, and $\boldsymbol{A}$ is an AR coefficient matrix. Thus the image prior probability density function (PDF) may be written as

$\mathrm{p}\left(\boldsymbol{f}_{+} \mid \boldsymbol{a}, \boldsymbol{\sigma}_{v}\right)=\mathcal{N}\left(\boldsymbol{f}_{+} \mid \mathbf{0}, \boldsymbol{\Sigma}_{f}\right)$,

where $\quad \boldsymbol{\Sigma}_{f}=\mathbb{E}\left[\boldsymbol{f}_{+} \boldsymbol{f}_{+}^{T}\right]=(\boldsymbol{I}-\boldsymbol{A})^{-1} \boldsymbol{Q}_{v}(\boldsymbol{I}-\boldsymbol{A})^{-T}$.

With the SAR model, we denote $\boldsymbol{C}=\boldsymbol{I}-\boldsymbol{A}$, and a first order non-causal AR model with coefficients 0.25 , representing the discrete Laplacian, is used to define $\boldsymbol{A}$. ${ }^{1}$ With this notation we have

$$
\mathrm{p}\left(\boldsymbol{f}_{+} \mid \boldsymbol{\sigma}_{v}\right) \propto \operatorname{det}\left|\boldsymbol{Q}_{v}\right|^{-\frac{1}{2}} \exp \left[-\frac{1}{2} \boldsymbol{f}_{+}^{T} \boldsymbol{C}^{T} \boldsymbol{Q}_{v}^{-1} \boldsymbol{C}_{+}\right] .
$$

Now we may segment the canvas $\mathcal{R}_{+}$into $R$ blocks or regions, $\mathcal{R}_{r} \subset \mathcal{R}_{+}$, of size $L_{r}$ pixels, and denote the pixels bordering $\mathcal{R}_{r}$ by $\mathcal{R}_{r b}$, such that $\mathcal{R}_{r_{+}}=\mathcal{R}_{r} \cup \mathcal{R}_{r b}$ (see Fig. 1 b)). For a given region $\mathcal{R}_{r}$, the image values inside it are denoted $f_{r}$, while those at its exterior border as $\boldsymbol{f}_{r b}$ (these pixels are in $\mathcal{R}_{r_{+}}$but not $\mathcal{R}_{r}$ ).

The excitation signal in $\mathcal{R}_{r}$ may then be written $\boldsymbol{v}_{r}=\boldsymbol{C}_{r} \boldsymbol{f}_{r}+$ $\boldsymbol{C}_{r b} \boldsymbol{f}_{r b}=\boldsymbol{C}_{r_{+}} \boldsymbol{f}_{r_{+}}$, with appropriately defined matrices $\boldsymbol{C}_{r}, \boldsymbol{C}_{r b}$, and $\boldsymbol{C}_{r_{+}}$extracted from $\boldsymbol{C}$. If the variance of each block is $\sigma_{v_{r}}^{2}$, then $\boldsymbol{\sigma}_{v}=\left[\sigma_{v_{1}}^{2} \cdots \sigma_{v_{R}}^{2}\right]^{T}$ and $\boldsymbol{Q}_{v}=\operatorname{diag}\left(\left[\sigma_{v_{1}}^{2} \mathbf{1}_{L_{1}}^{T} \cdots \sigma_{v_{R}}^{2} \mathbf{1}_{L_{R}}^{T}\right]^{T}\right) ; \mathbf{1}_{L}$ is a vector of $L$ ones. Now we may write

$$
\mathrm{p}\left(\boldsymbol{f}_{+} \mid \boldsymbol{\sigma}_{v}\right) \propto \prod_{r=1}^{R} \sigma_{v_{r}}^{-L_{r}} \exp \left[-\frac{1}{2} \sigma_{v_{r}}^{-2} \boldsymbol{v}_{r}^{T} \boldsymbol{v}_{r}\right]
$$

Note that the while the excitation signal $v$ is independent in each region, the image in each region depends on its neighbours.

The blur model is similar to the image prior, but with a stationary covariance described by the discrete Laplacian operator $\boldsymbol{C}_{h}$, i.e. $\mathrm{p}\left(\boldsymbol{h} \mid \delta_{h}\right)=\mathcal{N}\left(\boldsymbol{h} \mid 0, \delta_{h}\left(\boldsymbol{C}_{h}^{T} \boldsymbol{C}_{h}\right)^{-1}\right)$, where $\delta_{h}$ controls the importance placed on the prior (see [2]).

\footnotetext{
${ }^{1}$ It should also be noted that the full Laplacian matrix $\boldsymbol{C}$ should be modified at the boundaries, when used in the spatial domain, to avoid the assumption that the image outside the extended $m_{+} \times n_{+}$region is zero [5]. This further reduces boundary artefacts.
}

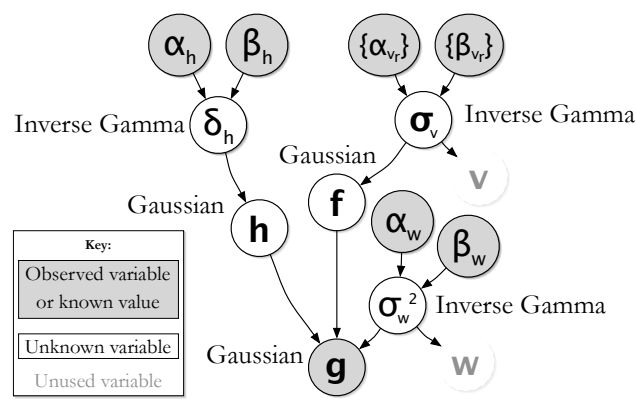

Fig. 2. Graphical model showing relationships between variables

\subsection{Hyperprior Models}

To remove dependance on exact specification of the prior models, whose hyperparameters are in practice unknown, the hyperpriors may be specified. A standard way to do this, resulting in more tractable solutions, is to use conjugate priors. For the hyperparameters, which are all variances of Gaussian distributions, the standard conjugate priors are inverse Gamma distributions; thus we have:

$$
\begin{aligned}
\mathrm{p}\left(\delta_{h}\right) & =\mathcal{I} \mathcal{G}\left(\delta_{h} \mid \alpha_{h}, \beta_{h}\right) \\
\mathrm{p}\left(\boldsymbol{\sigma}_{v}\right) & =\prod_{r=1}^{R} \mathcal{I} \mathcal{G}\left(\sigma_{v_{r}}^{2} \mid \alpha_{v_{r}}, \beta_{v_{r}}\right) \\
\mathrm{p}\left(\sigma_{w}^{2}\right) & =\mathcal{I} \mathcal{G}\left(\sigma_{w}^{2} \mid \alpha_{w}, \beta_{w}\right)
\end{aligned}
$$

where the inverse gamma distribution, defined for positive $\sigma^{2}$ is

$$
\mathcal{I} \mathcal{G}\left(\sigma^{2} \mid \alpha, \beta\right)=\frac{\beta^{\alpha}}{\Gamma(\alpha)} \sigma^{-2(\alpha+1)} e^{-\frac{\beta}{\sigma^{2}}},
$$

with $\quad \mathbb{E}\left[\sigma^{2}\right]=\frac{\beta}{\alpha-1}, \quad \operatorname{Var}\left[\sigma^{2}\right]=\frac{\beta^{2}}{(\alpha-1)^{2}(\alpha-2)}$

\section{VARIATIONAL BAYESIAN INFERENCE}

The complete model is shown using the directed acyclic graph (DAG), or Bayesian network, in Fig. 2 We denote the full set of unknowns as $\boldsymbol{\Theta}=\left\{\boldsymbol{f}_{+}, \boldsymbol{\sigma}_{v}, \boldsymbol{h}, \delta_{h}, \sigma_{w}^{2}\right\}$, and $\boldsymbol{\Theta}_{\backslash \boldsymbol{\theta}}$ this set omitting variable $\boldsymbol{\theta}$.

We wish to perform inference using the posterior distribution, $\mathrm{p}(\boldsymbol{\Theta} \mid \boldsymbol{g}) \propto \mathrm{p}(g \mid \boldsymbol{\Theta}) \mathrm{p}(\boldsymbol{\Theta})$. However for the model presented there is no analytic solution to maximise this distribution. Following the approach in [2], we derive a separable analytic approximation of the posterior as

$$
\mathrm{p}(\boldsymbol{\Theta} \mid \boldsymbol{g}) \approx \mathrm{q}(\boldsymbol{\Theta})=\prod_{\boldsymbol{\theta} \in \boldsymbol{\Theta}} \mathrm{q}(\boldsymbol{\theta}) .
$$

An iterative scheme is then used to estimate the optimum distributions for each variable $\boldsymbol{\theta}$.

We aim to make the approximation as good as possible by minimising the Kullback-Leibler (KL) divergence between the distributions:

$$
\begin{aligned}
& \mathrm{C}_{\mathrm{KL}}(\mathrm{q}(\boldsymbol{\Theta}) \| \mathrm{p}(\boldsymbol{\Theta} \mid \boldsymbol{g}))=\int_{\boldsymbol{\Theta}} \mathrm{q}(\boldsymbol{\Theta}) \log \left(\frac{\mathrm{q}(\boldsymbol{\Theta})}{\mathrm{p}(\boldsymbol{\Theta} \mid \boldsymbol{g})}\right) \mathrm{d} \boldsymbol{\Theta} \\
& =\int_{\boldsymbol{\Theta} \backslash \boldsymbol{\theta}} \mathrm{q}\left(\boldsymbol{\Theta}_{\backslash \boldsymbol{\theta}}\right) \int_{\boldsymbol{\theta}} \mathrm{q}(\boldsymbol{\theta}) \log \left(\frac{\mathrm{q}(\boldsymbol{\Theta} \backslash \boldsymbol{\theta}) \mathrm{q}(\boldsymbol{\theta})}{\mathrm{p}(\boldsymbol{g} \mid \boldsymbol{\Theta}) \mathrm{p}(\boldsymbol{\Theta})}\right) \mathrm{d} \boldsymbol{\theta} \mathrm{d} \boldsymbol{\Theta}_{\backslash \boldsymbol{\theta}}+\text { const. }
\end{aligned}
$$




$$
\begin{aligned}
& =\overbrace{\int_{\boldsymbol{\Theta} \backslash \boldsymbol{\theta}} \mathrm{q}\left(\boldsymbol{\Theta}_{\backslash \boldsymbol{\theta}}\right) \log \mathrm{q}\left(\boldsymbol{\Theta}_{\backslash \boldsymbol{\theta}}\right) \mathrm{d} \boldsymbol{\Theta}_{\backslash \boldsymbol{\theta}}}^{\text {independent of } \mathrm{q}(\boldsymbol{\theta})} \overbrace{\int_{\boldsymbol{\theta}} \mathrm{q}(\boldsymbol{\theta}) \mathrm{d} \boldsymbol{\theta}}^{=1}+\text { const. } \\
& +\int_{\boldsymbol{\Theta}_{\backslash \boldsymbol{\theta}}} \mathrm{q}\left(\boldsymbol{\Theta}_{\backslash \boldsymbol{\theta}}\right) \int_{\boldsymbol{\theta}} \mathrm{q}(\boldsymbol{\theta}) \log \left(\frac{\mathrm{q}(\boldsymbol{\theta})}{\mathrm{p}(\boldsymbol{g} \mid \boldsymbol{\Theta}) \mathrm{p}(\boldsymbol{\Theta})}\right) \mathrm{d} \boldsymbol{\theta} \mathrm{d} \boldsymbol{\Theta}_{\backslash \boldsymbol{\theta}}
\end{aligned}
$$

This rearrangement is due to the separable approximation. To obtain the optimal distribution $\hat{\mathrm{q}}(\Theta)$, we find for each $\boldsymbol{\theta}$ :

$$
\begin{aligned}
& \hat{\mathrm{q}}(\boldsymbol{\theta})=\underset{\mathrm{q}(\boldsymbol{\theta})}{\operatorname{argmin}} \mathrm{C}_{\mathrm{KL}}(\mathrm{q}(\boldsymbol{\Theta}) \| \mathrm{p}(\boldsymbol{\Theta} \mid \boldsymbol{g})) \\
& =\underset{\mathrm{q}(\boldsymbol{\theta})}{\operatorname{argmin}} \mathbb{E}\left[\int_{\boldsymbol{\theta}} \mathrm{q}(\boldsymbol{\theta}) \log \left(\frac{\mathrm{q}(\boldsymbol{\theta})}{\mathrm{p}(\boldsymbol{g} \mid \boldsymbol{\Theta}) \mathrm{p}(\boldsymbol{\Theta})}\right) \mathrm{d} \boldsymbol{\theta}\right]_{\mathrm{q}\left(\boldsymbol{\Theta}_{\backslash \boldsymbol{\theta}}\right)}
\end{aligned}
$$

where any terms independent of $\mathrm{q}(\boldsymbol{\theta})$ may be treated as constants. Differentiating the argument with respect to $\mathrm{q}(\boldsymbol{\theta})$ gives

$$
\log \hat{\mathrm{q}}(\boldsymbol{\theta})=\text { const. }+\mathbb{E}[\log (\mathrm{p}(\boldsymbol{g} \mid \boldsymbol{\Theta}) \mathrm{p}(\boldsymbol{\Theta}))]_{\mathrm{q}\left(\boldsymbol{\Theta}_{\backslash \boldsymbol{\theta}}\right)}
$$

The constant appears under the constraint $\int \hat{\mathrm{q}}(\boldsymbol{\theta}) \mathrm{d} \boldsymbol{\theta}=1$.

\section{DISTRIBUTION APPROXIMATIONS}

Given the approximation in (13, the distributions may be calculated using (18). For the distribution $\hat{\mathrm{q}}\left(\boldsymbol{\sigma}_{v}\right)$, using (18) (again ignoring terms independent of $\boldsymbol{\sigma}_{v}$ ), we find:

$$
\begin{aligned}
& \log \hat{\mathrm{q}}\left(\boldsymbol{\sigma}_{v}\right) \propto \mathbb{E}\left[\log \left(\mathrm{p}\left(\boldsymbol{f} \mid \boldsymbol{\sigma}_{v}\right) \mathrm{p}\left(\boldsymbol{\sigma}_{v}\right)\right)\right]_{\mathrm{q}\left(\boldsymbol{\Theta}_{\backslash \boldsymbol{\sigma}_{v}}\right)} \\
& \propto \log \left(\mathrm{p}\left(\boldsymbol{\sigma}_{v}\right)\right)-\frac{1}{2} \sum_{r=1}^{R} L_{r} \log \left(\sigma_{v_{r}}^{2}\right)+\mathbb{E}\left[\sigma_{v_{r}}^{-2} \boldsymbol{v}_{r}^{T} \boldsymbol{v}_{r}\right]_{\mathrm{q}\left(\boldsymbol{f}_{+}\right)} \\
& \Rightarrow \hat{\mathrm{q}}\left(\boldsymbol{\sigma}_{v}\right)=\prod_{r=1}^{R} \mathcal{I} \mathcal{G}\left(\sigma_{v_{r}}^{2} \mid \alpha_{v_{r}}+\frac{L_{r}}{2}, \beta_{v_{r}}+\frac{1}{2} \mathbb{E}\left[\boldsymbol{v}_{r}^{T} \boldsymbol{v}_{r}\right]_{\mathrm{q}\left(\boldsymbol{f}_{+}\right)}\right)
\end{aligned}
$$

This is in the form of a product of independent inverse Gamma distributions, over the variance of each region.

We may then use the iterative VB (IVB) approach to estimate the optimal distributions from the data; at each iteration, $k$, we denote these by $\mathrm{q}^{k}(\boldsymbol{\theta})$. This amounts to updating each $\mathrm{q}^{k}(\boldsymbol{\theta})$ in turn, using the expectations of the other previously found q distributions.

Intuitively, all we need to update each $\mathrm{q}^{k}(\boldsymbol{\theta})$ are its moments; since the the first two moments completely characterise the Gaussian and inverse Gamma distributions we are concerned with, these are all we need calculate to update the other distributions. We use $\mathbb{E}^{k}[\boldsymbol{\theta}]$ to denote the expectation of $\boldsymbol{\theta}$ with respect to the distribution $\mathrm{q}^{k}(\boldsymbol{\theta})$, and $\operatorname{cov}^{k}[\boldsymbol{\theta}]$ the covariance of $\boldsymbol{\theta}$ at iteration $k$.

Observe that $\mathrm{q}^{k}\left(\sigma_{v_{r}}^{2}\right)$ in 21] is in the form $\mathcal{I} \mathcal{G}(\theta \mid \alpha, \beta)=$ $\mathcal{I} \mathcal{G}\left(\theta \mid \alpha_{\theta}+x, \beta_{\theta}+y\right) ; \alpha_{\theta}$ and $\beta_{\theta}$ come from the prior, whose mean $\frac{\beta_{\theta}}{\alpha_{\theta}-1}$ we will now denote by $\mathbb{E}^{0}[\theta]$. Then to find $\mathbb{E}^{k}\left[\sigma_{v_{r}}^{2}\right]$, we apply the following identity for an inverse-Gamma distribution:

$$
\begin{aligned}
\mathbb{E}[\theta] & =\frac{\beta}{\alpha-1}=\frac{\beta_{\theta}+y}{\alpha_{\theta}+x-1} \\
= & \underbrace{\left(\frac{\alpha_{\theta}-1}{\alpha_{\theta}+x-1}\right)}_{\gamma_{\theta}} \underbrace{\left(\frac{\beta_{\theta}}{\alpha_{\theta}-1}\right)}_{\mathbb{E}^{0}[\theta]}+\underbrace{\left(\frac{x}{\alpha_{\theta}+x-1}\right)}_{1-\gamma_{\theta}}\left(\frac{y}{x}\right)
\end{aligned}
$$

which is a combination of the prior mean and the term $\frac{y}{x}$ weighted by a confidence parameter, $\gamma_{\theta}$, which takes values between zero and one. Thus we may supply $\mathbb{E}^{0}[\theta]$ and $\gamma_{\theta}$ in place of $\alpha_{\theta}$ and $\beta_{\theta}$.

The expectation in $y$ must be evaluated; in the case of $\mathrm{q}^{k+1}\left(\sigma_{v_{r}}^{2}\right)$ :

$$
\begin{aligned}
\mathbb{E}\left[\boldsymbol{v}_{r}^{T} \boldsymbol{v}_{r}\right]_{\mathrm{q}^{k}\left(\boldsymbol{f}_{+}\right)}=\mathbb{E}\left[\left\|\boldsymbol{C}_{r_{+}} \boldsymbol{f}_{r_{+}}\right\|^{2}\right]_{\mathrm{q}^{k}\left(\boldsymbol{f}_{r_{+}}\right)} \\
=\mathbb{E}^{k}\left[\boldsymbol{v}_{r}\right]^{T} \mathbb{E}^{k}\left[\boldsymbol{v}_{r}\right]+\operatorname{tr}\left[\boldsymbol{C}_{r_{+}}^{T} \boldsymbol{C}_{r_{+}} \operatorname{cov}^{k}\left[\boldsymbol{f}_{r_{+}}\right]\right]
\end{aligned}
$$

$\mathbb{E}^{k}\left[\boldsymbol{v}_{r}\right]$ is found simply by extracting the relevant pixels from $\mathbb{E}^{k}[\boldsymbol{v}]=$ $\boldsymbol{C} \mathbb{E}^{k}\left[\boldsymbol{f}_{+}\right]$. Note that 25 is derived using the identities $\boldsymbol{x}^{T} \boldsymbol{x}=$ $\operatorname{tr}\left[\boldsymbol{x} \boldsymbol{x}^{T}\right]$ and $\mathbb{E}\left[\boldsymbol{x} \boldsymbol{x}^{T}\right]=\mathbb{E}[\boldsymbol{x}] \mathbb{E}[\boldsymbol{x}]^{T}+\operatorname{cov}[\boldsymbol{x}]$.

The distributions and moments for the variables $\boldsymbol{f}_{+}, \boldsymbol{h}, \delta_{h}, \sigma_{w}^{2}$ are found in a similar manner using (18); the derivations are as for the corresponding variables in [2]: $\hat{\mathrm{q}}\left(\boldsymbol{f}_{+}\right)$and $\hat{\mathrm{q}}(\boldsymbol{h})$ are Gaussian, and $\hat{\mathrm{q}}\left(\delta_{h}\right)$ and $\hat{\mathrm{q}}\left(\sigma_{w}^{2}\right)$ are inverse Gamma distributions. Their use in the IVB procedure is described in Algorithm 1

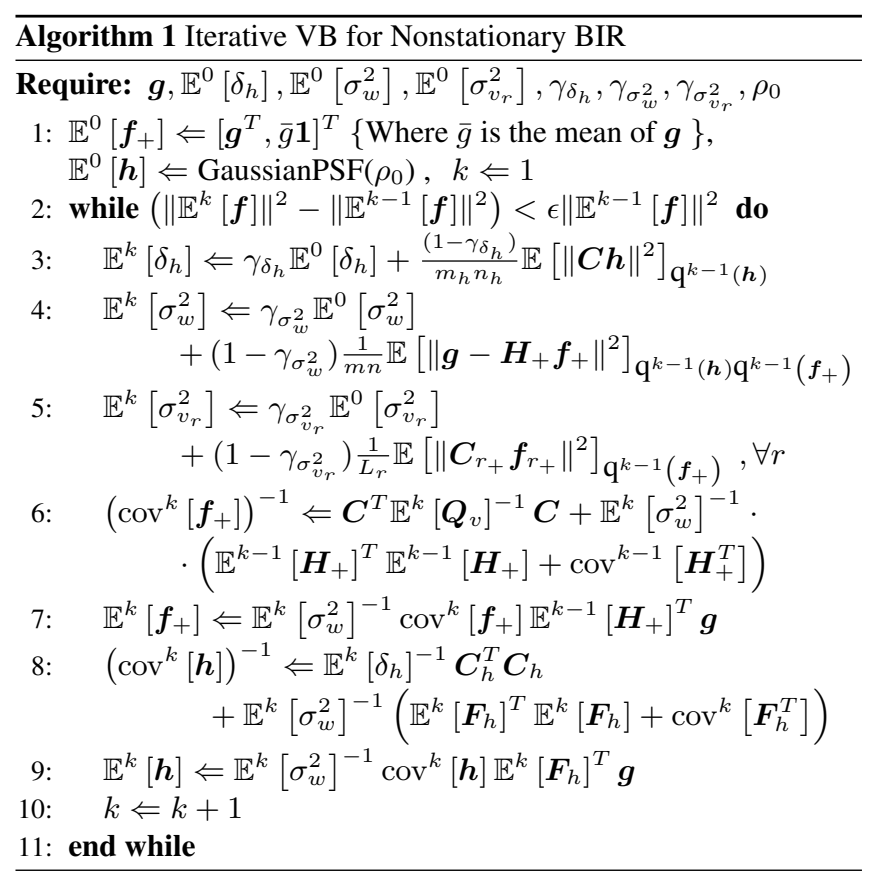

\section{SPATIAL DOMAIN IMPLEMENTATION}

In practice, to implement in the spatial domain, the matrices $\operatorname{cov}^{k}[\boldsymbol{h}]$ and $\operatorname{cov}^{k}\left[\boldsymbol{f}_{+}\right]$may be too large to invert. We have chosen to approximate the distributions $\mathrm{q}\left(\boldsymbol{f}_{+}\right)$and $\mathrm{q}(\boldsymbol{h})$ as degenerate, i.e. point estimates, and hence these terms, and the terms $\operatorname{cov}^{k}\left[\boldsymbol{F}_{h}^{T}\right]$ and $\operatorname{cov}^{k}\left[\boldsymbol{H}_{+}^{T}\right]$ which depend on them, are ignored in Algorithm 1 This enables us to use the conjugate gradients least sqaures (CGLS) algorithm [5] to efficiently solve the required linear equations for $\boldsymbol{f}_{+}$and $\boldsymbol{h}$. The downside with this approximation is that the hyperparameter estimation may be less reliable at low SNRs.

CGLS solves a least squares system

$$
\boldsymbol{M}^{T} \boldsymbol{M} \boldsymbol{x}=\boldsymbol{M}^{T} \boldsymbol{y}
$$

via an iterative procedure in which it is not required to calculate the product $\boldsymbol{M}^{T} \boldsymbol{M}$. In order to solve for $\boldsymbol{x}=\mathbb{E}^{k}\left[\boldsymbol{f}_{+}\right]$using this procedure, let $\boldsymbol{Q}_{v}^{-1}=\boldsymbol{L}^{T} \boldsymbol{L}, \boldsymbol{M}=\left[\frac{\boldsymbol{H}_{+}}{\boldsymbol{L} \boldsymbol{C}}\right]$, and $\boldsymbol{y}=\left[\frac{\boldsymbol{g}}{\mathbf{0}}\right]$. The solution for $\mathbb{E}^{k}[\boldsymbol{h}]$ is found similarly. 


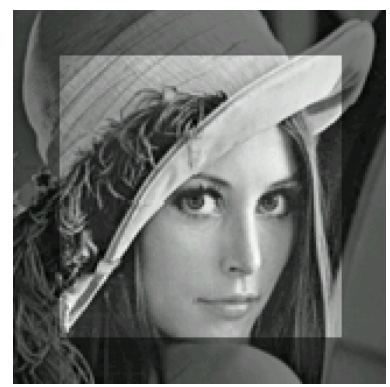

(a) $\boldsymbol{f}_{+}$

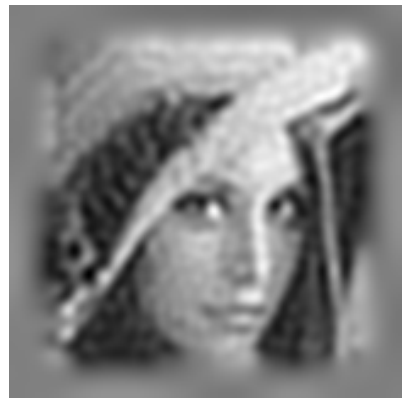

(c)

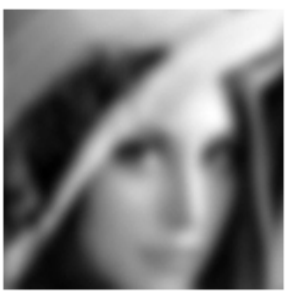

(b) $g$

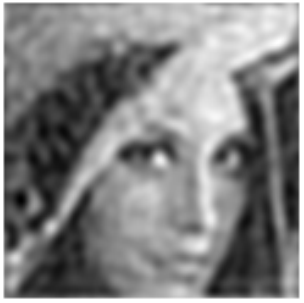

(d)
Fig. 3. (a) Original image, with extended canvas shown; (b) Blurred observation from the extended model; (c) Restoration using the proposed method (d) Restoration using the method in [2]

\section{EXPERIMENTAL RESULTS}

The proposed spatial domain restoration procedure has been tested and compared with the stationary solution found using the FFT, used in [2]. The Lena image shown in Fig. 3(a) is used for the original image, with the highlighted central region forming the observation region. This is blurred with a Gaussian PSF of variance $\rho=9$ pixels, and WGN with $\sigma_{w}^{2}=0.57$ added, giving the signal-to-noise ratio (SNR) of the blurred image, shown in Fig. 3(b) as 40dB.

For the restoration using the method in [2], the central portion, $\boldsymbol{f}$, of Fig. 3(a) is convolved with $\boldsymbol{h}$ using periodic boundary conditions, to give an observation $g$ consistent with the deblurring model (note that the FFT based algorithm is unable to work with a realistically blurred image due to the boundary effects).

In the proposed method, 10 CGLS iterations are performed at each VB iteration $k$, using $\mathbb{E}^{k-1}\left[\boldsymbol{f}_{+}\right]$and $\mathbb{E}^{k-1}[\boldsymbol{h}]$ for the initial guess in each case. It was found experimentally that both better numerical stability and speed are obtained if this is reduced to just 5 CGLS iterations per VB iteration, after the initial settling period where the noise variance $\sigma_{w}^{2}$ is estimated (this converges to a steady value after about 30 iterations).

The restored images found using each method after 384 iterations (using the termination threshold $\epsilon=10^{-4}$ ) are shown in Fig. 3(c) for the proposed method and Fig. 3(d) for the method in [2]. The same initial conditions for the hyperparameters are used: $\mathbb{E}^{0}\left[\delta_{h}\right]=$ $10^{-8} ; \mathbb{E}^{0}\left[\sigma_{w}^{2}\right]=0.5 ; \mathbb{E}^{0}\left[\sigma_{v_{r}}^{2}\right]=50, r \in \mathcal{R} ; \mathbb{E}^{0}\left[\sigma_{v_{r}}^{2}\right]=5, r \in$ $\mathcal{R}_{0} ; \gamma_{\delta_{h}}=0.5 ; \gamma_{\sigma_{w}^{2}}=0.5 ; \gamma_{\sigma_{v_{r}}^{2}}=0.7 ; \rho_{0}=1.0$. The proposed method used a fixed segmentation of regular $4 \times 4$ pixel blocks.

The ISNR defined as $10 \log _{10}\left(\|\boldsymbol{f}-\boldsymbol{g}\|^{2} /\left\|\boldsymbol{f}-\mathbb{E}^{k}[\boldsymbol{f}]\right\|^{2}\right)$ is found for the proposed method as $2.469 \mathrm{~dB}$ and $1.934 \mathrm{~dB}$ for the method in [2]. To make this evaluation fair and ignore the different

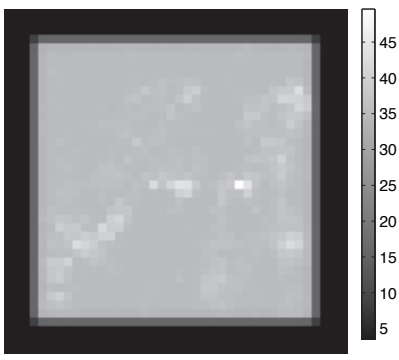

(a)

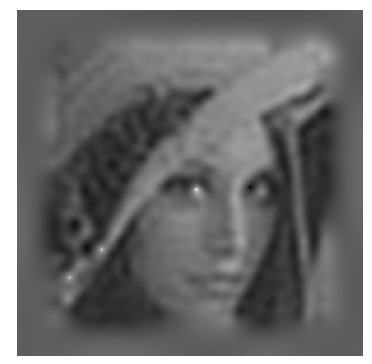

(b)
Fig. 4. (a) Local image variances $\sigma_{v_{r}}^{2}$;(b) Unstable restoration

boundary effects, the norms were calculated across a region slightly inset from the borders of $f$.

The estimated parameters are found as $\mathbb{E}\left[\delta_{h}\right]=5.5036 \times 10^{-9}$, $\mathbb{E}\left[\sigma_{w}^{2}\right]=0.3975, \mathbb{E}\left[\sigma_{v}^{2}\right]=35.965$ for the method in [2], and $\mathbb{E}\left[\delta_{h}\right]=5.6039 \times 10^{-9}, \mathbb{E}\left[\sigma_{w}^{2}\right]=0.3983$ for the proposed method. The values for $\mathbb{E}\left[\sigma_{v_{r}}^{2}\right]$ were in the range $33.703-50.681$ and are shown in Fig. 4(a)

As a further experiment, we observe that if the size of each region is reduced to one pixel, we obtain the image model proposed in [4]. A restoration using the same parameters above is shown in Fig. 4(b) the result is after 415 iterations. The ISNR is $2.3948 \mathrm{~dB}$. An interesting phenomena is observed: some of the variances $\sigma_{v_{r}}^{2}$ experience positive feedback as the iterations progress. When larger regions are used, this effect is mitigated. This suggests investigating other nonstationary models to gain better insight into the problem.

\section{CONCLUSIONS}

The VB approach for blind deconvolution has been extended to a spatial domain model which includes realistic modelling of boundary effects. This spatial domain model allows for nonstationary restorations to be performed, although the exact choice of model should be investigated further to obtain the best results.

\section{REFERENCES}

[1] T. E. Bishop, S. D. Babacan, B. Amizic, T. Chan, R. Molina, and A. K. Katsaggelos, "Blind image deconvolution: problem formulation and existing approaches," in Blind image deconvolution: Theory and Applications, P. Campisi and K. Egiazarian, Eds. CRC, 2007.

[2] R. Molina, J. Mateos, and A.K. Katsaggelos, "Blind deconvolution using a variational approach to parameter, image, and blur estimation," IEEE Trans Image Process, vol. 15, no. 12, pp. 3715-3727, 2006.

[3] T. E. Bishop and J. R. Hopgood, "Blind image restoration using a block-stationary signal model," in Proceedings 2006 IEEE International Conference on Acoustics, Speech and Signal Processing., May 2006, vol. 2, pp. II-853-II-856.

[4] G. Chantas, N. P. Galatsanos, and A. Likas, "Non stationary bayesian image restoration," in Proceedings - International Conference on Pattern Recognition, Cambridge, United Kingdom, 2004, vol. 4, pp. $689-692$.

[5] D. Calvetti and E. Somersalo, "Statistical elimination of boundary artefacts in image deblurring," Inverse Probl, vol. 21, no. 5, pp. 1697-1714, 2005. 